\title{
Behavioral Manipulation of Retrieval in a Spatial Memory Task for Drosophila melanogaster
}

\author{
Gerold Wustmann and Martin Heisenberg ${ }^{1}$ \\ Theodor-Boveri-Institut für Biowissenschaften (Biozentrum) \\ Lehrstuhl für Genetik Am Hubland \\ D-97074 Würzburg, Germany
}

\begin{abstract}
A paradigm for operant conditioning of freely walking single Drosopbila flies has been described previously. A fly can be conditioned to avoid one side of a small test chamber if the chamber is heated whenever the fly enters this side. In a subsequent memory test without heat the fly continues to avoid the previously heat-associated side. In this experimental design one cannot exclude that flies mark the heated side by an odor that they subsequently avoid during the test. As a final proof for associative learning in the present experiment, flies are trained in one chamber and tested for learning in another, similar one. Handling in the transfer experiment interferes with memory display, even if the fly is returned to the old chamber instead of a new one. Memory can be reactivated, however, by subjecting the fly to an additional brief training (priming), which is too short to establish significant learning in naive flies.

For efficient priming, heat has to be applied to the same side as during training in the old chamber. Only then the fly subsequently shows a side preference and avoids the side of the new chamber, which in the old one had been associated with heat. The two chambers are similar but not identical. The transfer experiment therefore raises the question as to what the flies use as spatial reference during training and test. In the light, they can be shown to orientate according to visual landmarks associated with the chamber. In complete darkness,
\end{abstract}

${ }^{1}$ Corresponding author. where training and memory scores do not differ from those in the light, they are assumed to use a combination of tactile and idiothetic information for orienting.

\section{Introduction}

Progress in the genetics of learning and memory in Drosophila melanogaster has been considerable in the last two decades (summarized, e.g., in Dudai 1988; Heisenberg 1989; DeZazzo and Tully 1995; Spatz 1995; Davis 1996). However, the field suffers from an important bias: Learning/ memory mutants have been screened only in paradigms for olfactory learning (Dudai et al. 1976; Quinn et al. 1979; Aceves-Pina et al. 1983; Dura et al. 1993), and most published mutant studies of learning and memory were carried out with classical olfactory conditioning (e.g., Tully and Quinn 1985; Dura et al. 1993).Knowledge concerning the molecular underpinnings of other types of associative learning, such as visual classical conditioning, or operant conditioning is still limited. A genetic dissection of different types of learning may broaden our understanding of its molecular, cellular, and behavioral basis.

A prerequisite for a genetic dissection is an efficient mutant screen. Although many learning paradigms have been developed for $D$. melanogaster, most of them are time-consuming and require considerable experience. Moreover, no appropiate screening procedure for operant conditioning is available so far. To overcome these shortcomings, Wustmann et al. (1996) developed a new paradigm for operant conditioning of freely walking flies. Individual animals are trained to avoid one side of a small test chamber. Whenever they enter this side, the chamber is heated. In a subsequent memory test without heat, the flies continue to avoid the

LEARNING \& MEMORY 4:328-336 @ 1997 by Cold Spring Harbor Laboratory Press ISSN1072-0502/97 \$5.00

$$
\begin{array}{llllllllllllllll}
L & E & A & R & N & I & N & G & \boldsymbol{Q} \\
\mathbf{3 2 8} & & & & & & & & & & & & & & &
\end{array}
$$


side previously associated with heat. The learning mutants dunce, rutabaga, and amnesiac avoid the heat-associated side but show no corresponding after effect (for dunce and rutabaga, Wustmann et al. 1996; for amnesiac, G. Wustmann, unpubl.). These and other observations suggest that the apparatus measures learning and memory. However, definite proof that the heating chamber scores associative learning is still missing. As an alternative interpretation, flies might deposite a repellant on one side of the chamber in response to heat and later avoid that odor without remembering anything.

In the present study we provide this proof. In addition, we determine the relevant cues that the fly associates with heat. The experiments reveal that the fly uses primarily visual landmarks in the light and tactile and idiothetic (Mittelstaedt and Mittelstaedt 1973) information in complete darkness. References outside the chamber, such as the electromagnetic field are ignored.

\section{Materials and Methods}

FLIES

Canton-S (CS) and Berlin wild-type strains were used. All flies tested were between 2 and 6 days old and were maintained at $25^{\circ} \mathrm{C}$ on standard cornmeal/molasses medium in a 16-hr light/8-hr dark cycle at $60 \%$ humidity. Females as well as males were studied ( $\sim 50 \%$ each $)$. No differences in the behavior of the two genders in the apparatus were detected, and the respective data were pooled.

\section{CONDITIONING APPARATUS}

The original apparatus developed by Wustmann et al. (1996) was used with slight modifications. The test apparatus (Fig. 1) is a rectangular chamber $(40 \times 4 \times 2.5 \mathrm{~mm})$. The upper and lower surfaces consist of Peltier elements, the sides of transparent acryl glass plates. A control circuit and a thermosensor in the chamber keep the Peltier elements at a permissive cold or restrictive bot temperature. The chamber is virtually subdivided perpendicular to its long axis into two halves (left and right) by a directionally selective light gate that informs the computer about whether the fly is in the left or right half. The light gate consists of two infrared (IR)-emitting diodes and two IR-sensitive photodetectors. The detectors are $3 \mathrm{~mm}$ apart

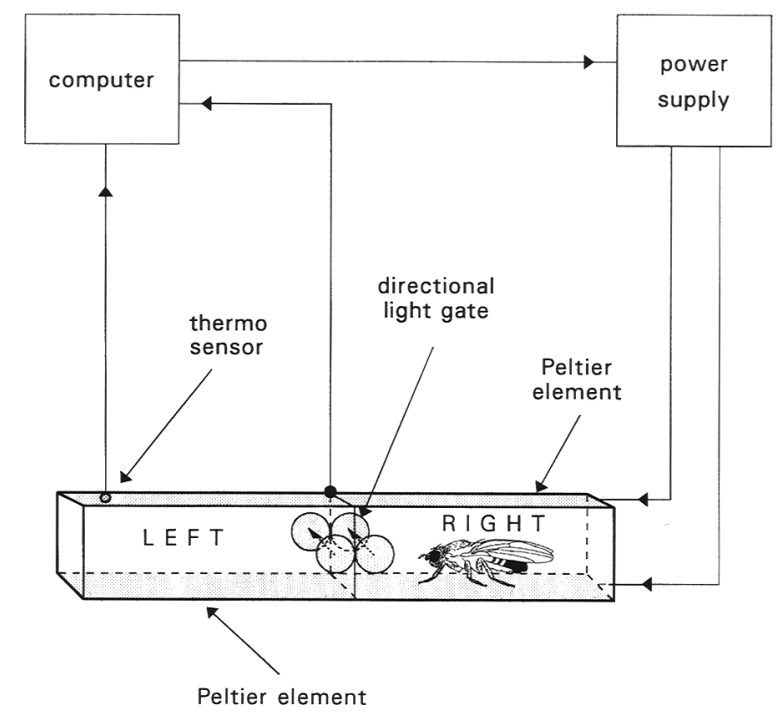

Figure 1: Heating chamber for operant conditioning. For explanations, see text. Adapted from Wustmann et al. (1996).

(center to center). The chamber is illuminated by four small lamps close to the corners of the alley; two are yellow, and two are blue. Looking from the position of the light gate down the alley in either direction, the blue lamps are always on the right side, and the yellow ones are on the left of the chamber's long axis. Whenever the fly is on the right side of the light gate only the two blue lamps are switched on, if the fly is on the left side, only the two yellow lights illuminate the chamber. A rack with 15 similar chambers was used for the experiments. Therefore, 15 flies were tested in parallel in the standard experiment (Figs. 2 and 9, below). In experiments involving transfer from one chamber to another, only two chambers were used in parallel in order to keep time between training and test short.

\section{PROCEDURES}

The experiments consist of three phases: (1) a preference test; (2) training, and (3) memory test. A computer controls the experiments in the following manner: During all three phases it continuously monitors the time and directions of transitions at the light gate. During training, in addition, it turns heat on when the fly enters one side and turns it off when the fly leaves it again. Note that the Peltier elements cover the whole length of the chamber. This ensures that the fly does not expe-

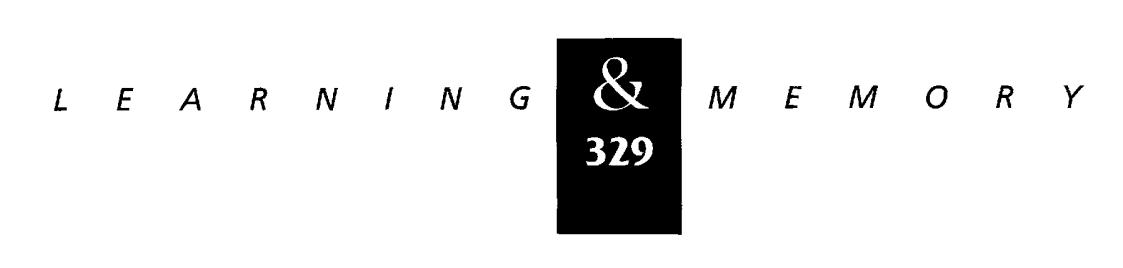


rience a spatial but only a temporal temperature gradient. bot is at $37^{\circ} \mathrm{C}\left( \pm 2^{\circ} \mathrm{C}\right)$ and cold at $25^{\circ} \mathrm{C}$ $\left( \pm 2^{\circ} \mathrm{C}\right)$. During the preference and memory tests the temperature is fixed at $25^{\circ} \mathrm{C}$ (cold), independently of the position of the fly. Between consecutive trials the sides for the permissive and restrictive conditions are switched. The chambers are used without cleaning for several hundred trials. After training, the memory test does not begin until the fly enters the other side; therefore, inactive flies are excluded. The activity of the flies is estimated by counting the number of passages at the light gate.

\section{ANALYSIS OF DATA}

The performance of individual flies is calculated as Performance Index $(\mathrm{PI})=(A-\mathrm{B}) /(A+B)$, with $A$ indicating the time during the training that the fly spends on the side associated with permissive temperature (cold) and B indicating the time on the bot side. The PI can vary between -1 and +1. A PI of zero indicates that the fly spends $50 \%$ of the time on the bot side. In the preference test, PI measures the fly's spontaneous preference for one side of the chamber; during training it indicates heat avoidance, and during the memory test it is a memory score. In the experiments half of the flies are conditioned to avoid left and the other half to avoid right. In this way the effects of spontaneous preferences for a distinct side and of slight assymmetries in the apparatus are largely eliminated from the data.

\section{STATISTICS}

Wilcoxon tests for significance of differences from zero (the random choice level) are carried out. The results are presented together with the standard error of the mean (s.E.M.).

\section{Results}

\section{PROOF FOR ASSOCIATIVE LEARNING}

A fly can be conditioned to avoid one side of the chamber if it is heated whenever the fly enters that side. In a subsequent memory test without heat, wild-type Drosophila continues to avoid the side associated previously with heat (Fig. 2). The question arises whether learning is involved in this behavior. An alternative hypothesis is that the animal deposits olfactory landmarks during the training and subsequently uses them to avoid the previously heated side during the so-called memory test.

To distinguish between these possibilities, we carried out a transfer experiment. Flies are trained in one chamber and tested in similar one in which sides could not be marked by odor landmarks. After a 30-sec preference test and a subsequent 3-min training, flies are gently aspirated into a pipette and transferred to a new, identical chamber where the 60 -sec memory test is carried out. The results are presented in Figure 3. Flies do not avoid the corresponding side in the new chamber. There are several explanations for this failure: They might be disorientated because they are missing the odor cues that guided them in the original experiment; the new chamber might not be sufficiently similar to the first one; or the transfer process might interfere with the memory test.

To examine the influence of the transfer on the behavior of the flies, individuals are taken out of the chamber after training and reintroduced into the same chamber for the memory test. Again, no memory score is obtained (Fig. 4). This suggests that handling disturbs the memory display. On the other hand, this does not rule out the possibility that the flies rely on deposited olfactory landmarks;

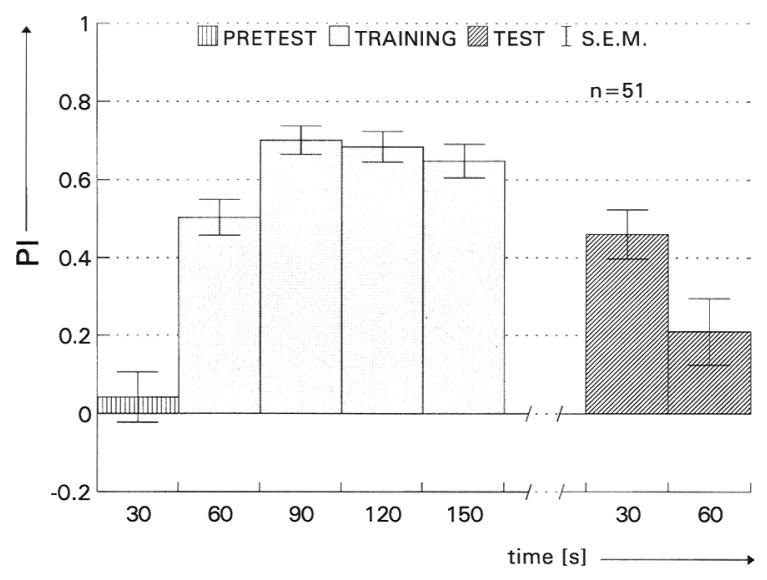

Figure 2: Average $\mathrm{Pl}$ before, during, and after avoidance training. The noxious stimulus is heat $\left(37 \pm 2^{\circ} \mathrm{C}\right)$. $\mathrm{PI}=(A-B) /(A+B)$, where $A$ is the time the fly spends on the side associated with no heat (cold) and $B$ the time on the heat-associated side (hot). A PI of zero indicates the random choice level. Flies are trained to avoid the left or the right side in an alternating sequence. (Wildtype CS flies; $n=51$.)

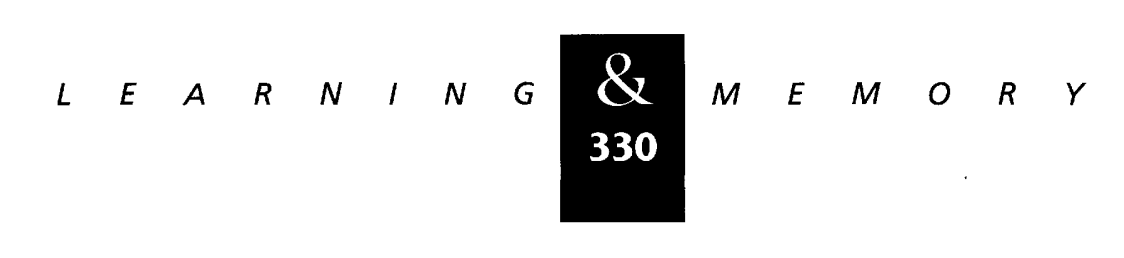




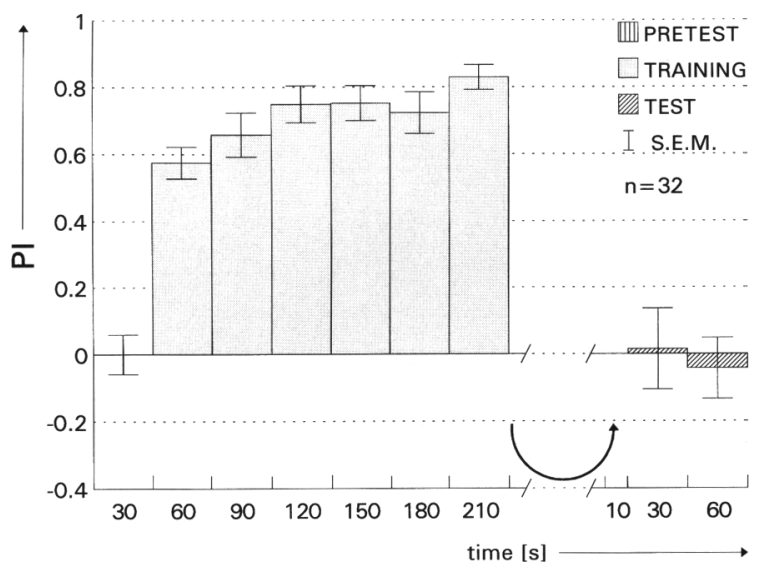

Figure 3: No memory in a transfer experiment. After a preference test flies are trained for $3 \mathrm{~min}$. Subsequently the animals are removed from the chamber and transferred to a similar one. (Wild-type Berlin flies; $n=32$.)

it simply shows that the transfer experiment is not suited to allow the fly to decide between the alternatives.

Assume that the animals fail to display the memory because they do not recognize the situation after transfer to be the same as that before. In this case, one could try to focus the fly's attention on the similarities between the old and new situation. To this end, flies are again transferred to a new chamber after the training phase. But, now, a 10-sec training session (priming) precedes the memory test. The heat-associated side is the same in both chambers. To assure that all flies experi-

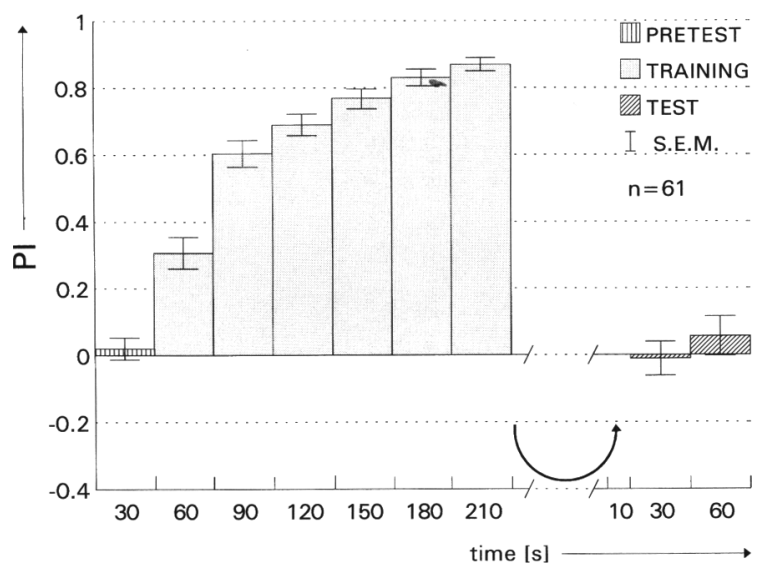

Figure 4: Transfer to the old chamber. After training flies (wild-type CS; $n=61$ ) are taken out of the chamber and reintroduced back into the same chamber for the memory test. The animals do not show any memory after handling between training and test. ence some heat at all during the 10 -sec period, priming is always started with the flies on the heatassociated side. The results are shown in Figure 5. After priming flies show a preference for the previously cold side. If flies are placed back into their old chamber and primed there, memory scores are not significantly greater (data not shown). As a further control, naive animals are treated for $10 \mathrm{sec}$ in the same manner (Fig. 6). This short training period alone does not establish a significant memory score in the subsequent test.

The experiments of Figures 5 and 6 suggest that the handling during the transfer to the new chamber does not destroy the memory trace but, rather, masks it. Yet, an alternative explanation still remains. The flies might be sensitized during training in the first chamber to quickly mark one side with olfactory landmarks during priming. In the second chamber, then, only the sensitized flies would be able to mark sufficiently the appropriate side during the 10-sec priming period. To test this hypothesis a so-called reverse-priming experiment is performed. Flies are trained for $3 \mathrm{~min}$ in one chamber. After transfer to the new chamber the alternative side of the chamber is punished during priming (compared to training in the first one). Flies trained to avoid the left side in the first chamber are primed to avoid the right side in the second one, and vice versa. If, in contrast to the naive flies,

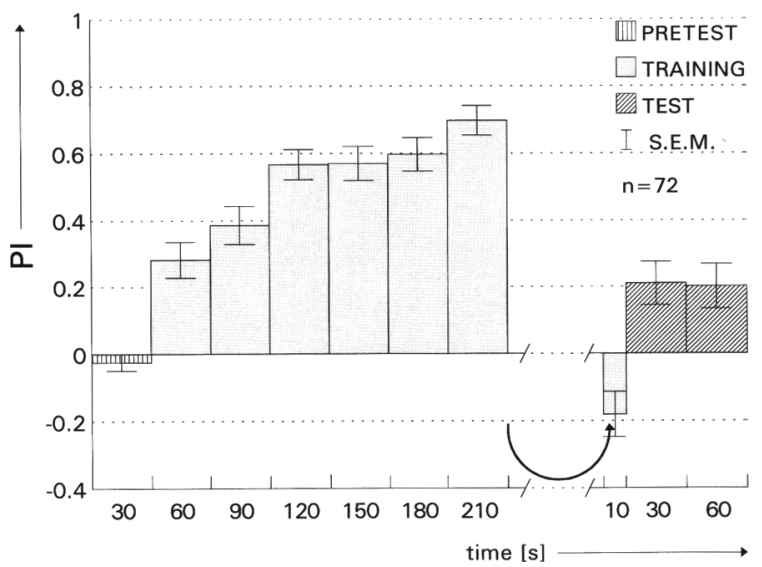

Figure 5: Priming experiment. Pls of preference test (30 $\mathrm{sec})$, training $(180 \mathrm{sec})$, priming $(10 \mathrm{sec})$ and memory test ( $60 \mathrm{sec}$ ). A fly (wild-type CS; $n=72$ ) is trained for 3 min in one chamber and is subsequently transferred to a new chamber. There, the priming (e.g., training) starts when the fly enters the punished side. Hence, each fly gets heated about $6 \mathrm{sec}$ during priming. After priming the memory test starts immediately. The PI of the learning test is significantly different from zero $(P<0.003)$.

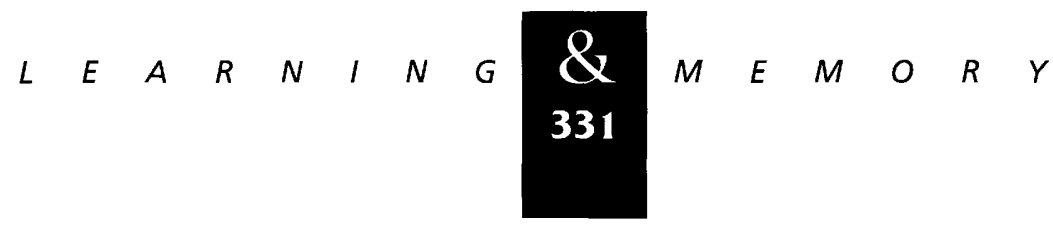




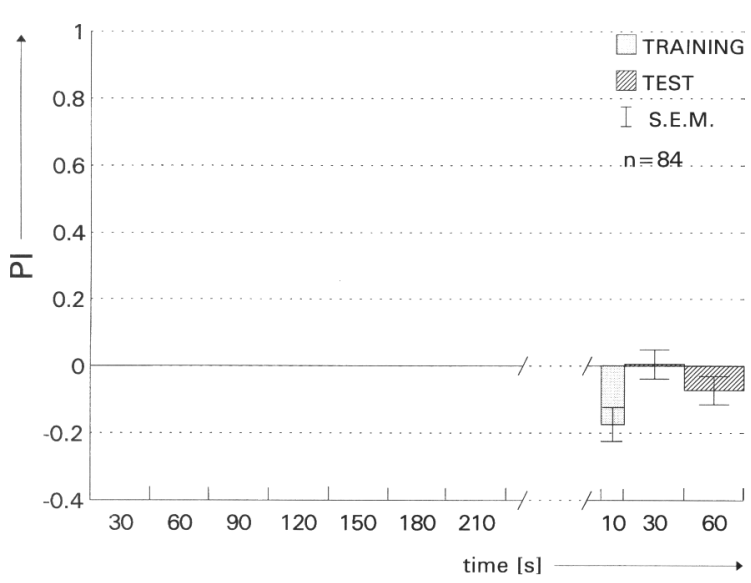

Figure 6: No learning is observed with priming alone. A fly (wild-type CS; $n=84$ ) is placed into a chamber and trained for $10 \mathrm{sec}$ with heat. Training begins when the fly enters the punished side. This is why the PI of the training is slightly negative. Immediately after training the test $(60 \mathrm{sec})$ starts.

these flies develop a side preference according to the polarity of priming, then during training in the old chamber they must have learned (non-associatively) to deposit during the short priming period olfactory landmarks for orientation in the memory test. The data (Fig. 7) reveal no such effect. Reverse priming seems to confuse the flies. They nei-

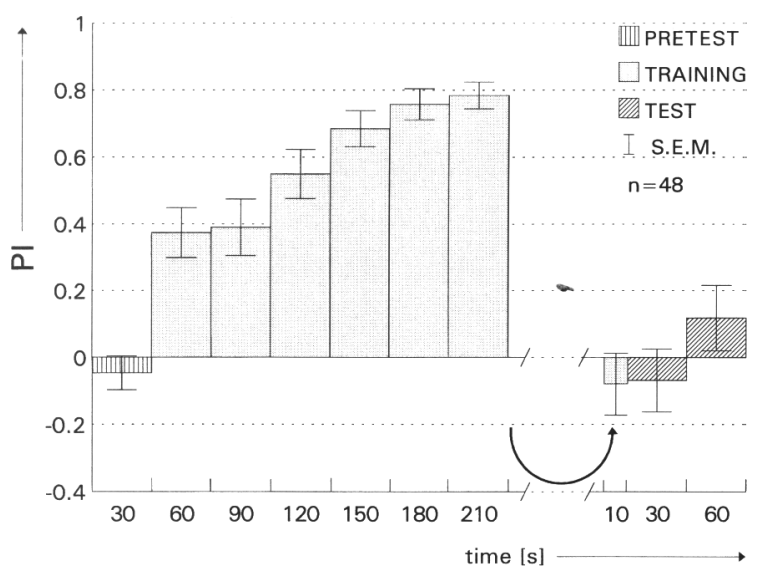

Figure 7: Priming of the alternative side. A fly (wildtype CS; $n=48$ ) is trained to avoid the left side in a chamber. Immediately after training the animal is transferred to another chamber. In the new one priming starts when the fly enters the punished side. But in the new chamber the fly is heated on the right side (the alternative side of the training in the former chamber). Half of the flies are trained right and primed left, the other half in the opposite way. No positive memory score is observed in the subsequent test. ther avoid the primed side nor the side corresponding to the one heated in the first chamber. Together, the two experiments indicate that the priming reactivates the memory of the training in the first chamber. Odor cues are not used as essential landmarks.

\section{SIDE OF ENTRY}

If the priming alone does not provide the reference for the choice between the two sides in the test, what are the cues telling the fly which side in the second chamber corresponds to which in the first one? In the experiments described so far flies are placed into the chambers always from the same side. It is possible, therefore, that flies use the side of entry as reference. To address this question, in the next transfer experiment flies are placed into and taken out of the training chamber from the left but are placed into the test chamber from the right. The results of Figure 8 show clearly that the animals avoid the previously punished side as well as they did when entering the two chambers from the same side. Obviously, they do not use the side of entry as a reference for orientation.

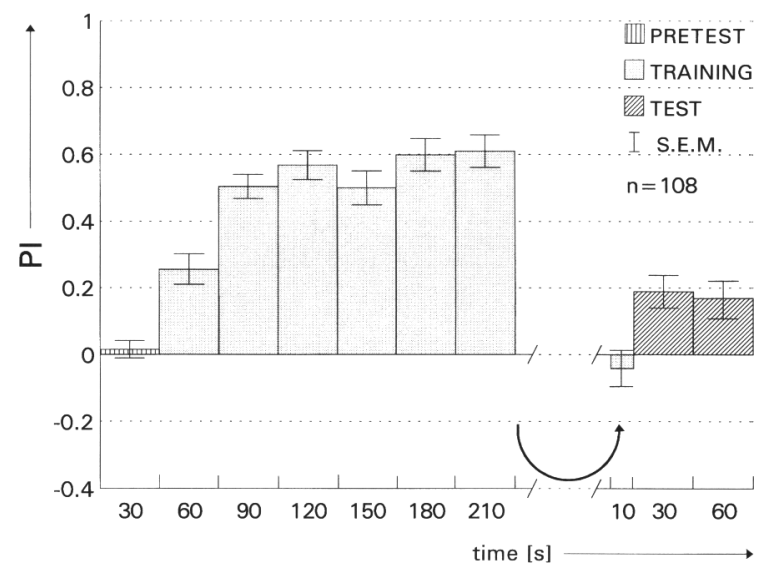

Figure 8: Transfer experiment with alternative entrances. A fly (CS; $n=108)$ is placed into a chamber from the left and trained to avoid one particular side (left or right). Immediately after training the fly is removed through the same left entrance and put into a new chamber through the other (right) entrance. The training in chamber 1 and priming in chamber 2 take place at the same side of the chambers. The PI of the memory test is significantly different from zero $(P<0.001)$. The flies do not use the entrance as a reference for orientation in the chambers.

$$
\text { n...... 㯰" }
$$




\section{LEARNING IN DARKNESS}

The previous experiment suggests that in the test chamber the flies can identify the sides corresponding to the heated and nonheated sides of the training chamber, irrespective of the side of entry and the side of priming. The chambers are illuminated through the lateral acryl glass plates by two blue or two yellow lights that are alternatively switched on depending on the fly's position in the chamber. In principle, the fly can distinguish its position with respect to the light gate by the direction and color of illumination. It is therefore conceivable that the flies use visual stimuli for their orientation in the chamber. To examine this hypothesis, the next experiments are conducted in complete darkness. After placing a fly into each chamber all lights are switched off. Only the two IR-emitting diodes of the directional light gate are still in operation, but flies are blind at this spectral wavelength $(\lambda=950 \mathrm{~nm}$ ) (Heisenberg and Wolf 1984). In Figure 9 the PIs in darkness are compared to those obtained under normal illumination conditions. There are no significant differences between the two groups. The data show clearly that no vision is required for this type of learning in the chamber.

In the present paradigm locomotor activity is a prerequisite for conditioning. Flies in darkness might still be less or more active than in the light.

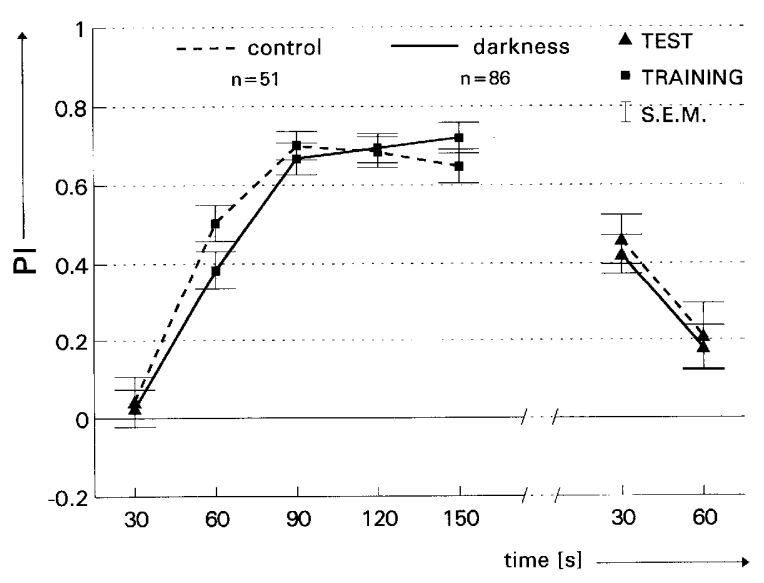

Figure 9: Learning in darkness. For one group of flies (CS; control; $n=51$; same data as Fig. 2) during the whole experiment the chambers and the laboratory are illuminated as during all others experiments described in Materials and Methods. For the other group of flies (CS; darkness; $n=86$ ) lights are switched off, i.e., flies are in complete darkness. No significant differences of the PIs between the two groups are seen.

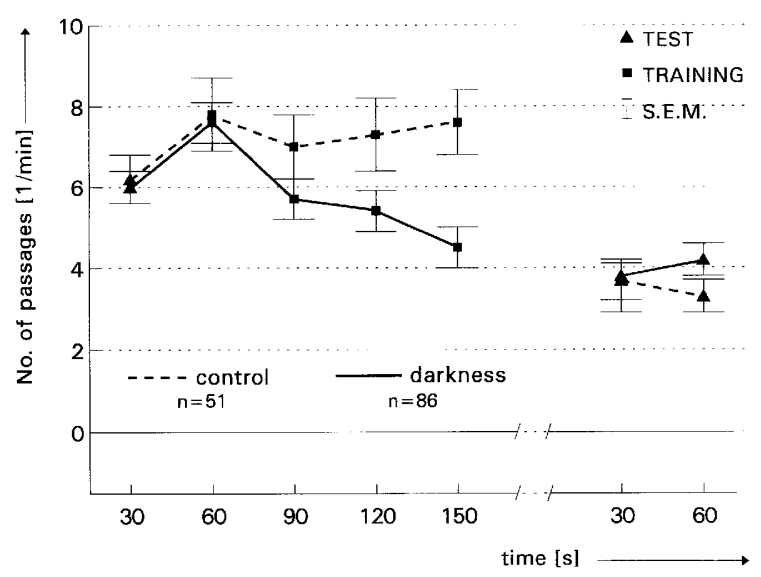

Figure 10: Average number of passages at the light gate per minute during the 240 -sec experimental session of the two groups of flies in the experiment of Fig. 9.

This would make the comparison of the two conditions more difficult. Activity scores are shown in Figure 10. During the first minute of the experiment no difference in locomotor activity (measured by counting the transitions at the light gate) is observed. In both groups a slight increase is seen after the beginning of the training period. Thereafter, the activity of the flies in darkness decreases during the rest of the training period, whereas it stays high in the light. Interestingly, during the transition between training and memory tests activity in the light decreases sharply to the level that both groups show during the memory tests. The same levels of activity during the memory tests in light and darkness support the above conclusion that light is not required for learning in the present experiment. The fly must use tactile and idiothetic information (Mittelstaedt and Mittelstaedt 1973) to distinguish between the two halves of the chamber.

\section{CUES FROM OUTSIDE THE CHAMBER}

In the transfer experiment, however, tactile and idiothetic information cannot provide the reference needed to match the two sides in the old and new chamber. If vision is not required, odor cues are not used and neither the side of entry nor the side of priming provides the reference in the test chamber; one wonders whether the electromagnetic field or other (unidentified) cues outside the chamber might serve that purpose. This hypothesis can be tested by a simple procedure: As above, flies are trained in one chamber and trans-

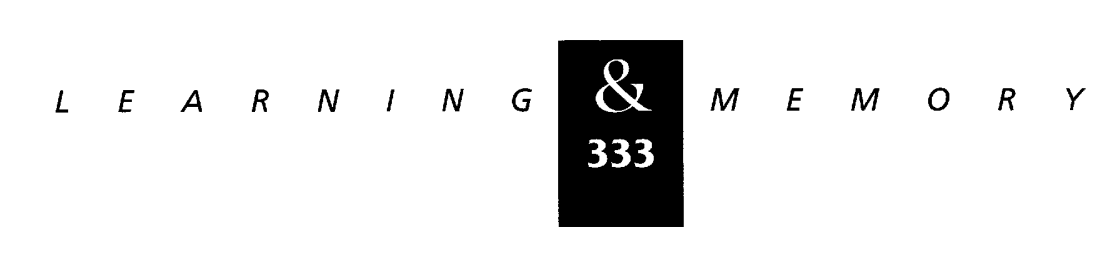


ferred for the test to another similar one. In the new chamber they are primed for $10 \mathrm{sec}$ and tested for $60 \mathrm{sec}$. In this case, however, the old and new chambers are orientated at right angles to each other. The positive memory score (Fig. 11) shows that the flies do not need any external reference such as the electromagnetic field to orientate correctly in the test chamber.

\section{WHAT IS THE REFERENCE?}

Have all conceivable references now been excluded? Do we have to postulate a new, yet unidentified sense for orientation in flies? No. The reader may be aware that we have demonstrated independence of vision only for the standard experiment without transfer. It is still possible that visual cues are used as reference in the test chamber after transfer. To test this last option flies are trained in one chamber in complete darkness and tested in similar chamber in darkness as well. Only during transfer the chambers are illuminated by the room light to facilitate the manipulations. In the two chambers opposite sides are heated, that is, the left side in chamber 1 and the right side for

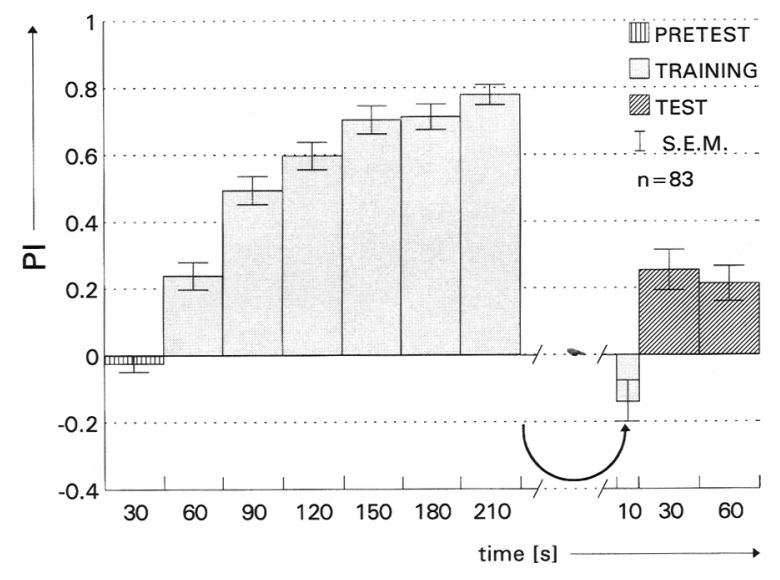

Figure 11: Test for outside references. Pls of preference test $(30 \mathrm{sec})$, training (180 sec), priming (10 sec), and memory test ( $60 \mathrm{sec}$ ). A fly (wild-type Berlin; $n=83$ ) is trained for $3 \mathrm{~min}$ in one chamber and is subsequently transferred to a similar one. The chambers are orientated at right angles to each other. Because of the $90^{\circ}$ rotation of any outside references between training and test, the flies should be unable to use them for avoiding the previously heated side. The PI of the memory test is significantly different from zero $(P<0.0001)$ and not different from that of the transfer experiment without rotation $(P>0.54)$.

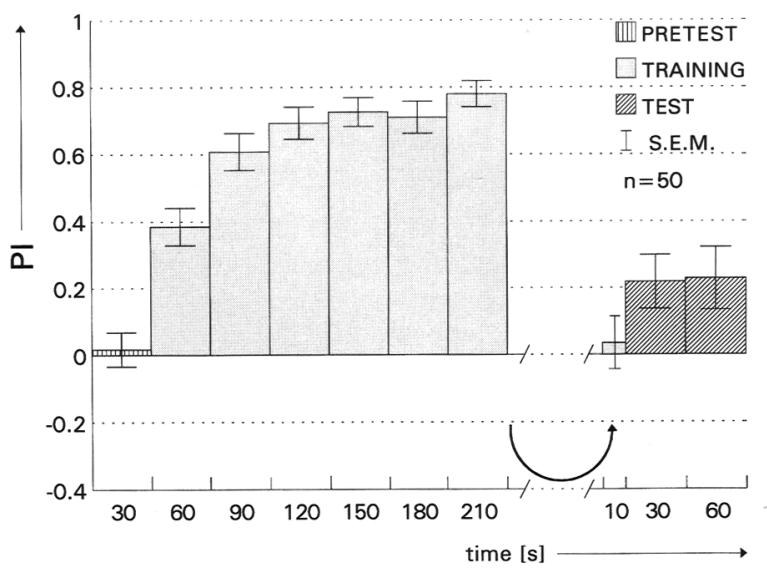

Figure 12: Learning in darkness: Priming of the alternative side. Flies (CS; $n=50$ ) are trained to avoid one particular side of chamber 1 . Immediately after training flies are transferred to chamber 2. In this chamber the flies are punished (priming) on the opposite side (compared to chamber 1$)$. In the test, animals keep avoiding the primed side $(P<0.006)$.

priming in chamber 2 , and vice versa. The results are shown in Figure 12. In contrast to the result in the light (Fig. 7), the flies do not show confusion but avoid the primed side. As in the light (Fig. 6), in darkness a 10-sec training session does not elicit a memory score in naive flies (Fig. 13). In darkness, the flies use the side of priming as reference. The different outcome of the experiment in the light (Fig. 7) shows that the flies do rely on visual cues in the transfer experiment if they are available.

\section{Discussion}

The heating chamber described by Wustmann et al. (1996) introduces a fast, simple, and robust single-fly memory test for Drosophila wild type and mutants. In the original presentation of the chamber the claim that it assesses learning and memory was based on circumstantial evidence. It could still be argued that the flies might deposit a chemical marker during training on one side of the chamber and later use it for proper orientation in the test, without any learning being involved. The transfer experiment in the present study firmly rules out this explanation.

Of particular interest is the finding that the simple transfer experiment does not work. A zero memory score is measured after the handling, irrespective of whether the fly is transferred to a new chamber or back into the old one. This result alone

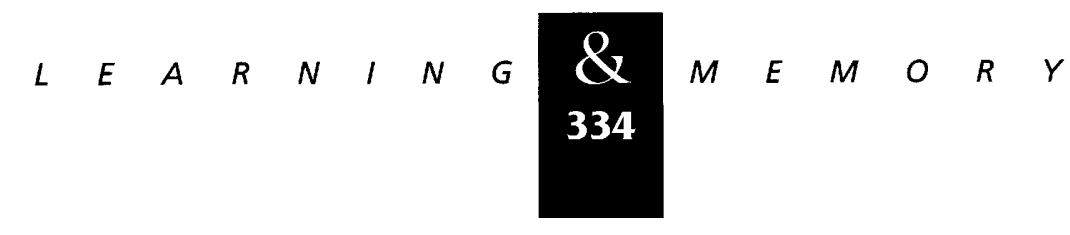




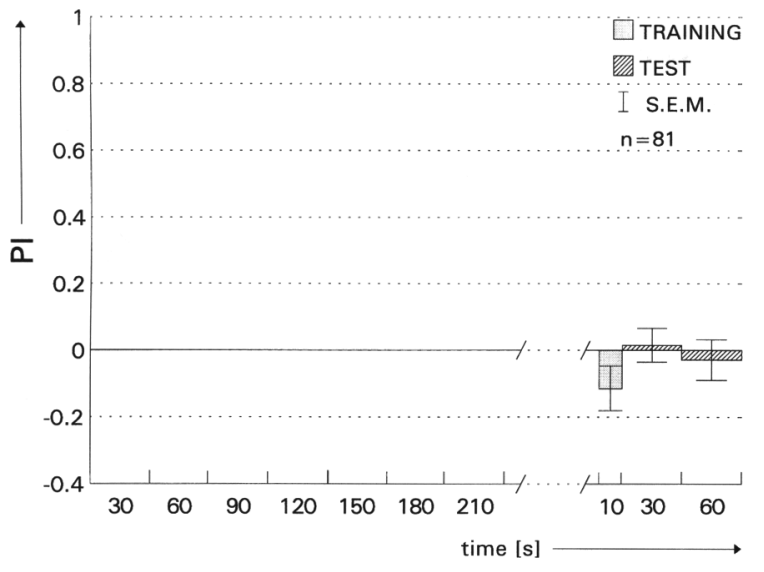

Figure 13: Learning in darkness: No learning is observed with priming alone. A fly (CS; $n=81$ ) is placed into a chamber and trained for $10 \mathrm{sec}$ with heat. Immediately after training the test $(60 \mathrm{sec})$ starts. No side preference is seen after the training.

might be interpreted to indicate that the handling erases the memory trace. However, with a brief training after the handling the PI is up again.

The priming has two independent effects: (1) It provides information about the polarity of the new chamber with respect to heat and lack of heat; and (2) it seems to channel the fly's "attention" to this particular task. The precise nature of the channeling is not understood. Attention has been described for Drosophila earlier in a visual orientation task in which animals confine their course control responses to certain regions of the visual field (Wolf and Heisenberg 1980; Heisenberg and Wolf 1984). It may be similar to the priming effect observed here in that the animal can selectively direct its behavior to certain aspects of the situation. In the present case, the memory of the conditioning process that is suppressed because of handling during the transfer is restored by a brief repetition of the original training procedure. That is, not the memory of the lesson but its retrieval is impaired in the present example. Impairment of retrieval as distinct from memory loss has also been reported for the Drosopbila mutant amnesiac (Quinn et al. 1979) and in honeybees (Menzel et al. 1991, Hammer and Menzel 1995). In the honey bees, injection of dopamine into the region of the central complex transiently interferes with memory retrieval. What is new in the present account is that retrieval is manipulated purely by behavioral means.

The argument inferring that the test PI in the transfer experiments reflects the reactivated memory from the training in the first chamber is based on two additional controls: (1) The priming in the test chamber by itself, that is, without the training in the first chamber, does not generate a positive PI (Fig. 6); and (2) with the normal illumination of the chambers, priming restores PIs only if heat is applied with the same polarity as during the training in the first chambers (Fig. 5). With the opposite polarity PIs are close to zero (Fig. 7) suggesting that the information about the polarity derived from the priming is in conflict with some other information about polarity stored in memory, which as we now know is visual (see below). This outcome implies that the side preference expressed by the test PI is at least partly based on information gathered in the first chamber and, consequently, cannot be explained entirely as an unconditional chemosensory response.

An important part of the conclusion is based on a comparison of the experiment of Figure 12 with that of Figure 7. In both cases, flies are trained in chamber 1 to avoid one particular side and primed in chamber 2 to avoid the opposite side. The only difference is the absence of light in the experiment of Figure 12. In this case, the animals rely entirely on the priming in their side preference. They avoid the primed side, although a 10sec training alone is not sufficient to induce a consistent side preference. Formally, the training in the first chamber could be said to increase the salience of the reinforcer (heat) during the subsequent priming. However, one should keep in mind that the fly, primarily, has to learn to treat the temporal sequence of heat and lack of heat as a spatial problem. Only after it has acquired this ability can it manage to get the polarity right during the 10 -sec priming period.

The experiment of Figure 7 indicates that the animals use visual cues, if present, as well as idiothetic and tactile information for their side preference. In darkness, they rely entirely on the latter. On the other hand, the additional visual cues do not increase the memory score if they have the same polarity as the priming. Little is known about what limits the PI during the test.

The transfer experiment should work only if the two chambers are recognized as similar by the flies. In fact, the flies do not indicate to us by their behavior that they distinguish them. After transfer to the new and to the old chamber they show the same memory and activity scores. This suggests that the two sides of the chamber also appear simi-

$$
\begin{array}{lllllllllllllllll}
\hline & E & A & R & N & I & N & G & \begin{array}{l}
\mathbf{Q} \\
335
\end{array} & M & E & M & O & R & Y
\end{array}
$$


lar to the flies. It is important to remember (see Materials and Methods) that the only visual cues the fly can exploit during the test are the color, subjective intensity, and direction of illumination, which change abruptly between two alternating states whenever the fly passes in front of the light gate. It is quite remarkable that the fly attributes any significance to this entirely artificial cue at all and that it relies on it about as much as on the 10 -sec priming to determine the heat/lack of heat polarity in the test chamber.

These experiments demonstrate that $D$. melanogaster is sufficiently well orientated in complete darkness to pass our avoidance and memory tests. This basic sense of space, which relies on idiothetic orientation and thus in turn only on tactile and proprioceptive information, is little understood in Drosophila as well as in humans.

\section{Acknowledgments}

The apparatus was built in the workshops of K. Öchsner and $\mathrm{H}$. Kaderschabeck. Their expertise substantially improved the electronic and mechanical design. The excellent technical assistance of S. Flurschütz is gratefully acknowledged. We also thank C. Hayward and J. Dickinson for working on the English of an earlier version of the manuscript, R. Wolf for redesigning Figure 1 , and $\mathrm{H}$. Spanheimer for final corrections on spelling.

The publication costs of this article were defrayed in part by payment of page charges. This article must therefore be hereby marked "advertisement" in accordance with 18 USC section 1734 solely to indicate this fact.

\section{References}

Aceves-Pina, E.O., R. Booker, J.S. Duerr, M.S. Livingstone, W.G. Quinn, R.F. Smith, P.P. Sziber, B.L. Tempel, and T.P. Tully. 1983. Learning and memory in Drosophila, studies with mutants. Cold Spring Harbor Symp. Quant. Biol. 48: 831-839.

Davis, R.L. 1996. Physiology and biochemistry of Drosophila learning mutants. Physiol. Rev. 76: 299-317.

DeZazzo, J. and T. Tully. 1995. Dissection of memory formation: From behavioral pharmacology to molecular genetics. Trends Neurosci. 18: 212-218.

Dudai, Y. 1988. Neurogenetic dissection of learning and short-term memory in Drosophila. Annu. Rev. Neurosci. 11: $537-563$.

Dudai, Y., Y. Jan, D. Byers, W.G. Quinn, and S. Benzer. 1976. dunce, a mutant of Drosophila deficient in learning. Proc. Natl. Acad. Sci. 73: 1684-1688.

Dura, J.-M., T. Preat, and T. Tully. 1993. Identification of linotte, a new gene affecting learning and memory in Drosophila melanogaster. J. Neurogenet. 9: 1-14.

Hammer, M. and R. Menzel. 1995. Learning and memory in the honeybee. J. Neurosci. 15: 1617-1630.

Heisenberg, M. 1989. Genetic approach to learning and memory (mnemogenetics) in Drosophila melanogaster. In Fundamentals of memory formation: Neural plasticity and brain function (ed. H. Rahmann), pp. 3-45. Gustav Fischer, Stuttgart, Germany.

Heisenberg, M. and R. Wolf. 1984. Vision in Drosophila. In Studies of brain function (ed. V. Braitenberg) Vol. 12. Springer, Berlin, Germany.

Menzel, R., M. Hammer, G. Braun, J. Mauelshagen, and M. Sugawa. 1991. Neurobiology of learning and memory in honeybees. In The behavior and physiology of bees (ed. L.J. Goodman, and R.C. Fisher), pp. 323-353. CAB International, Wallingford, UK.

Mittelstaedt, H. and M.-L. Mittelstaedt. 1973. Mechanismen der Orientierung ohne richtende Aussenreize. Fortschr. Zool. 21: 45-58.

Quinn, W.G., P.P. Sziber, and R. Booker. 1979. The Drosophila memory mutant amnesiac. Nature 277: 212-214.

Spatz, H.-Ch. 1995. Posttranslational modification of protein kinase A. The link between short-term and long-term memory. Behav. Brain Res. 66: 79-84.

Tully, T. and W.G. Quinn. 1985. Classical conditioning and retention in normal and mutant Drosophila melanogaster. J. Comp. Physiol. A 156: 263-277.

Wolf, R. and M. Heisenberg. 1980. On the fine structure of yaw torque in visual flight orientation of Drosophila melanogaster. II. A temporally and spatially variable weighting function for the visual field ("visual attention"). J. Comp. Physiol. A 140: 69-80.

Wustmann, G., K. Rein, R. Wolf, and M. Heisenberg. 1996. A new paradigm for operant conditioning of Drosophila melanogaster. J. Comp. Physiol. A 179: 429-436.

Received June 23, 1997; accepted in revised form September 26, 1997.

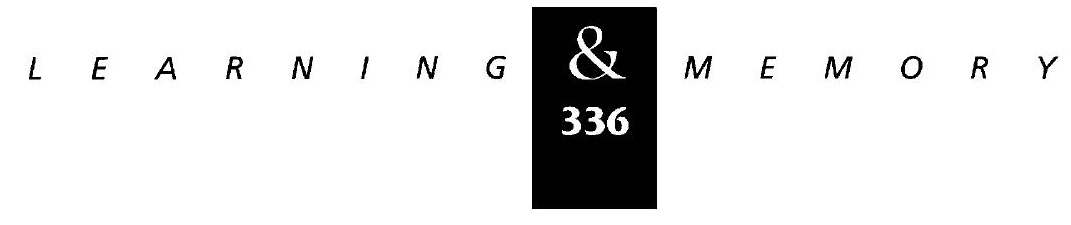




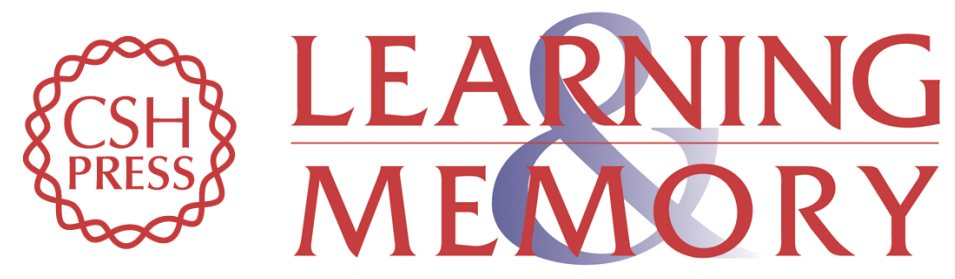

\section{Behavioral manipulation of retrieval in a spatial memory task for Drosophila melanogaster.}

G Wustmann and M Heisenberg

Learn. Mem. 1997, 4:

Access the most recent version at doi:10.1101//m.4.4.328

References This article cites 13 articles, 3 of which can be accessed free at: http://learnmem.cshlp.org/content/4/4/328.full.html\#ref-list-1

License

Email Alerting Receive free email alerts when new articles cite this article - sign up in the box at the Service top right corner of the article or click here. 